\title{
lincR-Ccr2-5'AS and THRIL as potential biomarkers of multiple sclerosis
}

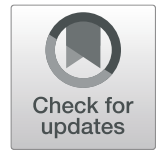

\author{
Olfat Gamil Shaker ${ }^{1}$, Amr Hassan², Asmaa Mohammed Mohammed ${ }^{3}$ and Shereen Rashad Mohammed ${ }^{3 *}$
}

\begin{abstract}
Background: Multiple sclerosis (MS) is a demyelinating disease affecting the central nervous system (CNS). Long non-coding RNAs (IncRNAs) were believed to play a role in the pathogenesis of neurological disorders including MS. lincR-Ccr2-5'AS is expressed in the Thelper2 (Th2) lineage. TNF-a heterogeneous nuclear ribonucleoprotein L (THRIL) causes the induction of TNF- $a$ and regulates innate immune response and inflammation. We investigated the expression of lincR-CCr2-5'AS and THRIL in MS to clarify their association with MS risk and the clinical features.

Results: LincR-Ccr2-5'AS was significantly downregulated in MS patients (fold change $=0.43 \pm 0.29, p=0.03$ ). The expression level was significantly low in patients with motor weakness and optic neuritis, patients with Expanded Disability Status Scale (EDSS) $\geq 5.5$, and treatment-naïve patients. THRIL was significantly upregulated in MS patients (fold change $=6.18 \pm 2, p=0.02$ ). Its expression was significantly higher in patients with relapsing-remitting multiple sclerosis (RRMS), patients with motor weakness, patients with EDSS $\leq 5$, and patients who received interferon.

Conclusion: Our results showed the downregulation of lincR-Ccr2-5'AS and the upregulation of IncRNA THRIL in MS patients. This differential expression of both IncRNAs may have an important role in MS pathogenesis.
\end{abstract}

Keywords: Multiple sclerosis, lincR-Ccr2-5'AS, IncRNA, THRIL

\section{Background}

Multiple sclerosis (MS) is an autoimmune demyelinating disease. It affects the central nervous system (CNS) and is marked by gliosis and degeneration of axons [1]. It attacks young adults, especially females. It has a variety of symptoms with variable severity $[2,3]$. Patients with MS start to present by a relapsing-remitting period in which there are sensory disorders, diplopia, and optic neuritis that become stable over time. However, continuing signs of CNS dysfunction become irreversible [3].

MS pathogenesis involves many genetic and environmental factors [4]. The advance of biomarkers involved in pathogenesis, response to the treatment, and prognosis of the disease could all lead to the optimal clinical management of the disease [5].

\footnotetext{
* Correspondence: shereenkaddafy@yahoo.com; Srk00@fayoum.edu.eg ${ }^{3}$ Department of Medical Biochemistry and Molecular Biology, Faculty of Medicine, Fayoum University, Fayoum, Egypt

Full list of author information is available at the end of the article
}

Non-coding RNAs (ncRNA) are implicated in human physiology and pathology. Growing evidence suggests a key regulatory role played by ncRNA, especially long non-coding RNA [6]. They are considered an essential regulator of the biological processes, regulating "chromatin remodeling," "gene transcription," and "protein transport" [7]. Recently, IncRNAs are believed to have an essential role in the pathogenesis of many neurological diseases. Few studies investigated the potential role of lncRNAs in MS pathology [8]. LncRNAs are dysregulated in MS patients, especially in peripheral blood mononuclear cells, which propose their enrollment in MS pathogenesis [9].

The exact pathophysiology for the occurrence of MS is still unidentified. It was shown that $\mathrm{T}$ helper cells are implicated in MS pathogenesis [10]. The $\mathrm{T}$ helper2 (Th2) lineage shows an expression of the lincR-Ccr2-5' AS, which in return is linked to GATA-binding protein 3 (GATA3), the "master regulator" of Th2 [11]. Diminution of this lncRNA decreases the expression of 
chemokine genes in the Th2 lineage [11]. These chemokines contribute to the enrollment of $\mathrm{T}$ lymphocytes and macrophages into the CNS, which is believed to be involved in the pathogenesis of MS [12].

Tumor necrosis factor-alpha (TNF- $\alpha$ ) is a cytokine that plays a key role in many autoimmune diseases, such as rheumatoid arthritis (RA) and multiple sclerosis (MS) [13]. It modulates the initiation of the inflammatory process and keeps it all through [13]. Many systemic and local stimuli are involved in the production of TNF- $\alpha$ [14]. THRIL is a lncRNA related to innate immunity. It combines with heterogeneous ribonucleoprotein particle (hnRNPs) which binds to promoter/enhancer regions of the TNF- $\alpha$ gene and causes stimulation of transcription and an elevation in TNF- $\alpha$ gene expression [11]. Levels of TNF- $\alpha$ are significantly elevated in cerebrospinal fluid (CSF) and plasma of MS patients. Besides, they are elevated in CSF of RRMS and secondary progressive MS (SPMS) patients [15].

lincR-Ccr2-5' AS and THRIL have been recently described to be key regulatory molecules sharing a vital role in regulating the immune response. Accordingly, the abnormal expression of lncRNAs has been proposed as an underlying cause of MS pathogenesis.

Given the regulatory role of lincR-Ccr2-5'AS and THRIL in the immune response in MS, we hypothesized that such long non-coding RNA may affect the susceptibility to MS. We aimed to evaluate the relative expression levels of lincR-Ccr2-5'AS and THRIL in MS patients, and to study their potential association with the clinical presentation, disease severity, course, and progression of MS.

\section{Methods}

\section{Subjects}

This case-control study was conducted on one hundred and thirty-four subjects and was divided into two groups:

- Group I comprised seventy-four patients $(83.8 \%$ females, $16.2 \%$ males, mean age $32.5 \pm 8.1$ years) with MS: relapsing-remitting multiple sclerosis (RRMS) and secondary progressive multiple sclerosis (SPMS) fulfilling the Revised McDonald's criteria for the diagnosis of MS 2010 [16]. They were recruited from Kasr Al-Ainy Multiple Sclerosis Unit (KAMSU)", Cairo University Hospital, and outpatient clinics of the neurology department Fayoum University. We excluded pregnant females,; patients with a history of other autoimmune diseases, cancer, or chronic infectious disease, or patients with recent infection a month before the time of enrollment. Patients on steroid therapy were excluded as well. We recorded full history for all patients and examined them. We assessed the initial symptoms and presentation of MS. MS manifestations included optic neuritis; sensory symptoms as numbness, itching, or burning pain; or motor symptoms, such as weakness or ataxia. We evaluated the clinical disability using the Expanded Disability Status Scale (EDSS) at the time of patient enrollment. EDSS is the most commonly used scale in MS patients. EDSS, with a scoring system from 0 to 10 , reveals the patient's morbidity. While zeropoint is a normal neurological examination, 10 points show the MS-related death cases. The patients were classified according to EDSS into 3 groups, EDSS $=1.0-3.0$, EDSS $=3.5-5$, and $\mathrm{EDSS} \geq 5.5$ [17].

- Group II: The control group comprised sixty healthy volunteers, age and sex matched (80\% females, $20 \%$ males, mean age $30.2 \pm 9.2$ years), with no family history of MS or any other autoimmune disorders.

\section{Blood sample collection}

A vacutainer system was used for collecting blood samples $(5 \mathrm{ml})$ from all subjects involved in the study. Samples were collected in tubes with separator gels that lodge between packed cells and the top serum layer, permitted to clot for $15 \mathrm{~min}$, and then centrifuged at $4000 \times g$ for $10 \mathrm{~min}$. The serum samples were separated from clotted whole blood and stored at $-80{ }^{\circ} \mathrm{C}$ until use.

\section{RNA extraction}

The serum was separated for the quantitative expression of lncRNAs by real-time PCR. MiRNeasy extraction kit (Qiagen, Valencia, CA) was used to extract RNA using QIAzol lysis reagent. NanoDrop 2000 (Thermo scientific, USA) was used to determine the concentration of RNA.

\section{IncRNA expression by quantitative RT-PCR}

Sixty nanograms of RNA was used in the reverse transcription (RT) step. The final volume of RT reaction was $20 \mu \mathrm{l}$ using the RT2 first strand kit (Qiagen, Valencia, $\mathrm{CA}$ ) as stated by the manufacturer's directions. Complementary DNA (cDNA) was stored at $-80{ }^{\circ} \mathrm{C}$. GAPDH was used as an internal control for lncRNAs [18-20]. Readymade primers and Maxima SYBR Green PCR kit (Thermo, USA) were used for the assessment of lncRNA serum expression. Twenty-five-microliter reaction mixtures were used in RT-PCR. PCR conditions are as follows: $95{ }^{\circ} \mathrm{C}$ for $10 \mathrm{~min}$, followed by 45 cycles at $95{ }^{\circ} \mathrm{C}$ for $15 \mathrm{~s}$, and $60{ }^{\circ} \mathrm{C}$ for $60 \mathrm{~s}$. The gene expression related to the internal control $\left(2^{-\Delta \mathrm{Ct}}\right)$ was calculated. To ensure the specificity of the corresponding RT-PCR reactions, a melt curve analysis was done. The expression levels of the two target genes were calculated using the $2^{-\Delta \Delta C t}$ equation. The cycle threshold $(\mathrm{Ct})$ value is the number of $\mathrm{qPCR}$ cycles required for the fluorescent signal to pass a specified threshold. $\Delta \mathrm{Ct}$ was calculated by 
subtracting the $\mathrm{Ct}$ values of internal control from those of lincR-Ccr2-5'AS and THRIL. $\Delta \Delta$ Ct was calculated by subtracting the $\Delta \mathrm{Ct}$ of the control samples from the $\Delta \mathrm{Ct}$ of the patients' samples. For the control sample, $\Delta \Delta \mathrm{Ct}$ equals zero, and 20 equals one [21].

\section{Statistical analysis}

Data were collected, coded, and entered into Microsoft Access. They were analyzed by the SPSS software version 18 on Microsoft Windows 7. The statistical analysis included a simple descriptive represented by numbers and percentages for qualitative data, standard deviations as a measure of dispersion for quantitative parametric data, inferential statistic test, independent Student's $t$ test, one-way ANOVA test, Chi-square test, and Bivariate Pearson correlation test. The $p$ values were calculated based on a Student's $t$-test of the replicate $2^{\wedge}$ $(-\Delta \mathrm{CT})$ values for each gene in both the control and patient groups. Logistic analysis was performed to determine the disease predictors. The receiver operating characteristic (ROC) curve was conducted to exemplify the diagnostic power of both long non-coding RNAs. $P$ values less than 0.05 were considered significant.

\section{Results}

Demographic and clinical data are summarized in Table 1 Seventy-four patients with MS (RRMS and SPMS types) (83.8\% females, $16.2 \%$ males, mean age $32.5 \pm 8.1$ years) were enrolled in this study together with sixty controls. They did not significantly differ in age or sex distribution. Out of 74 patients, 54 patients (73\%) had RRMS and 20 patients (27\%) had SPMS. 24 patients (32.4\%) had optic neuritis, 10 patients (13.5\%) had sensory symptoms, while 40 patients $(54.1 \%)$ had motor symptoms. Four patients $(5.4 \%)$ had a positive family history. The

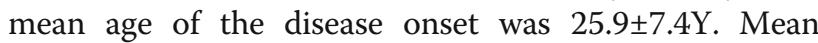
EDSS was $3.4 \pm 1.9$; 42 patients $(56.8 \%)$ had EDSS scores ranging from 1 to 3 and 14 patients (18.9\%) had EDSS scores ranging from 3.5 to 5 , while 18 patients (24.3\%) had EDSS scores $\geq 5.5$. Regarding treatment options, 40 patients $(54.1 \%)$ received interferon and 16 patients (21.6\%) received immunosuppressant in the form of

Table 1 Demographic and clinical characteristics of study groups

\begin{tabular}{|c|c|c|c|}
\hline & MS patients $(n=74)$ & Control $(n=60)$ & $p$-value \\
\hline Age [years] mean $\pm S D$ & $32.5 \pm 8.1$ & $30.2 \pm 9.2$ & 0.13 \\
\hline \multicolumn{4}{|l|}{ Sex [no. (\%)] } \\
\hline Female & $62(83.8 \%)$ & $48(80 \%)$ & 0.59 \\
\hline Male & $12(16.2 \%)$ & $12(20 \%)$ & \\
\hline \multicolumn{4}{|l|}{ Types of MS [no. (\%)] } \\
\hline RRMS & $54(73 \%)$ & & \\
\hline SPMS & $20(27 \%)$ & & \\
\hline \multicolumn{4}{|l|}{ Initial presentation [no. (\%)] } \\
\hline Optic neuritis & $24(32.4 \%)$ & & \\
\hline Sensory symptoms & $10(13.5 \%)$ & & \\
\hline Motor symptoms & $40(54.1 \%)$ & & \\
\hline \multicolumn{4}{|l|}{ Family history of MS [no. (\%)] } \\
\hline Positive & $4(5.4 \%)$ & 0 & \\
\hline Negative & 70 (94.6\%) & $60(100 \%)$ & \\
\hline Age of onset (years) [mean $\pm S D$ ] & $25.9 \pm 7.4$ & & \\
\hline EDSS score [mean $\pm S D$ ] & $3.4 \pm 1.9$ & & \\
\hline EDSS $=1-3$ & $42(56.8 \%)$ & & \\
\hline EDSS $=3.5-5$ & $14(18.9 \%)$ & & \\
\hline EDSS $\geq 5.5$ & $18(24.3 \%)$ & & \\
\hline \multicolumn{4}{|l|}{ Treatment [no. (\%)] } \\
\hline Treatment naive & $18(24.3 \%)$ & & \\
\hline Interferon & $40(54.1 \%)$ & & \\
\hline \multicolumn{4}{|l|}{ Immunosuppressants } \\
\hline Azathioprine & $12(16.2 \%)$ & & \\
\hline Cyclophosphamide & $4(5.4 \%)$ & & \\
\hline
\end{tabular}


azathioprine and cyclophosphamide, while 18 patients (24.3\%) were treatment naïve.

\section{Expression levels of lincR-Ccr2-5'AS and THRIL}

LincR-Ccr2-5'AS was found significantly downregulated in MS patients compared to controls; the decrease was $>2$-fold the controls, (fold change, $2^{-\Delta \Delta \mathrm{Ct}}=0.34, p=0.03$ ). On the other hand, IncRNA THRIL was found significantly upregulated in MS patients compared to controls with an increase $\geq 6$-fold the controls (fold change, $2^{-\Delta \Delta C t}$ $=6.18, p=0.02)$ (Figs. 1 and 2) (Table 2).

\section{The association between lincR-Ccr2-5'AS and THRIL} relative expression levels and patients' clinical characters Table 3 shows the relative expression levels of LincRCcr2-5' As and THRIL based on different clinical characters among cases. Regarding lincR-Ccr2-5'AS, there was no significant difference in relative expression levels between RRMS and SPMS patients. The relative expression level was significantly low in patients with motor weakness and optic neuritis compared to those with sensory symptoms $(p=0.001)$. Besides, patients with EDSS $\geq 5$ had significantly lower expression levels than those with EDSS $<5.5$ ( $p=0.001)$. There was a significantly low expression level among treatment-naïve patients and those on immunosuppressants $(p=0.001)$.

Regarding THRIL, patients with RRMS had significantly higher expression levels of THRIL as compared to those with SPMS $(p=0.04)$. Patients who initially had motor symptoms had a significantly higher expression level of THRIL compared to those with sensory and optic neuritis ( $p=0.001,0.05$ respectively). Patients with EDSS $<5$ had a significantly higher expression level of THRIL than those with EDSS $\geq 5.5$ ( $p=0.001)$. There was a significantly higher expression level among patients who received interferon compared to treatment-naïve patients and those on immunosuppressants $(p=0.001)$.

\section{Correlation between THRIL and lincR-Ccr2-5'AS with other variables among cases}

There was no statistically significant correlation between lincR-Ccr2-5'AS and THRIL expression levels. Moreover, there was no statistically significant correlation between the two IncRNAs and the age of patients, or the disease onset age (Table 4).

\section{ROC analysis to determine the diagnostic performance of} lincR-Ccr2-5'AS and THRIL

Serum expression levels of lincR-Ccr2-5'AS and THRIL had rational diagnostic performance. For lincR-Ccr2-5' AS, the AUC was $57.1 \%$, cutoff point $=0.49$, sensitivity $=71.9 \%$, specificity $=100 \%$, and accuracy $=85.9 \%$. For THRIL, the AUC was 0.615 , cutoff point $=1.024$, sensitivity $=57.1 \%$, specificity $=100 \%$, and accuracy $=78.6 \%$. (Fig. 3) (Table 5).

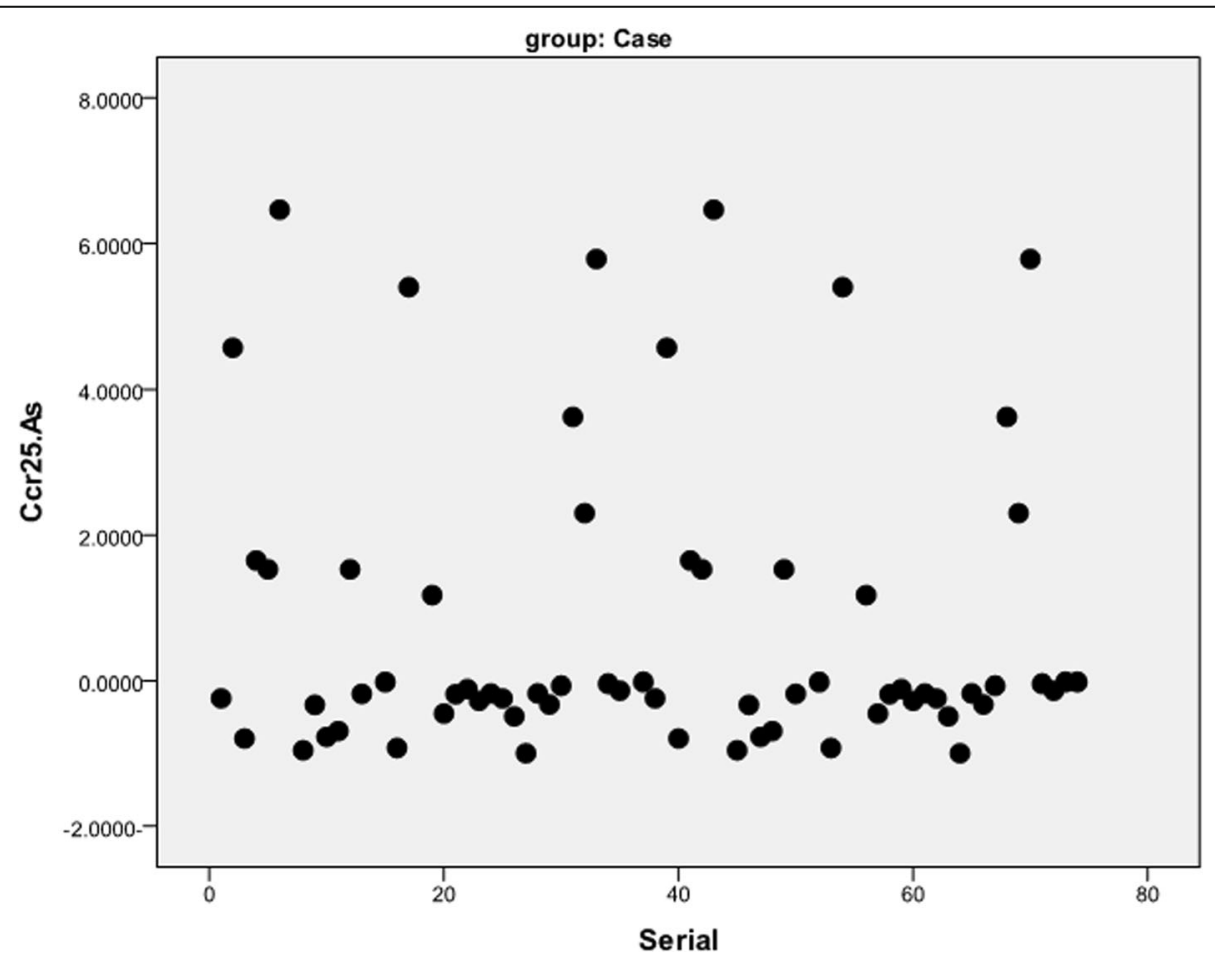

Fig. 1 Dot plot of lincR-Ccr2-5'AS fold change in MS patients 


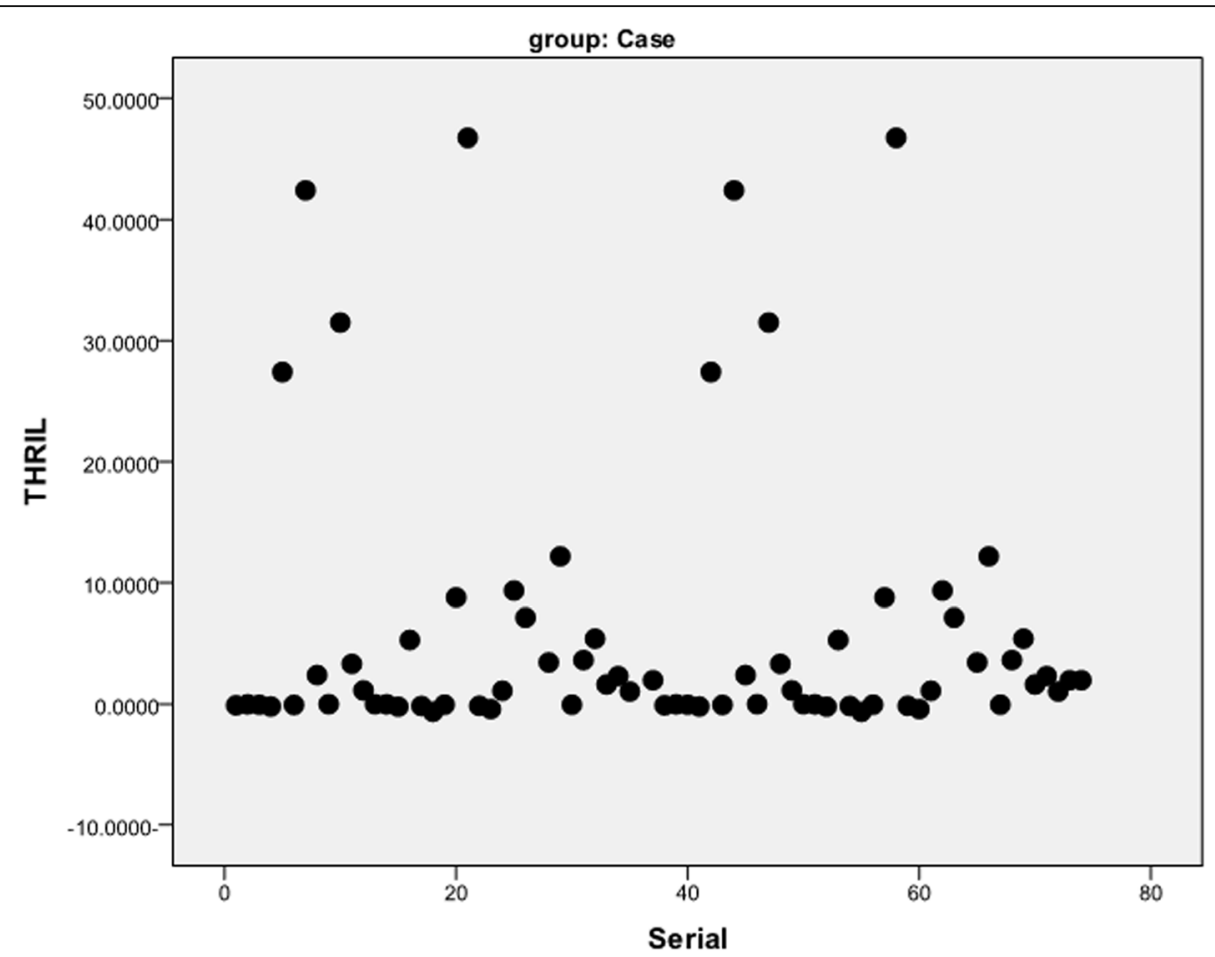

Fig. 2 Dot plot of THRIL fold change in MS patients

\section{Logistical regression analysis}

Binary logistical regression analyses were performed to predict the long non-coding RNA associated with an increased risk of multiple sclerosis (Table 6). THRIL was found to be a predictor of MS with a $p$-value $<0.05$ and $\mathrm{OR}=6.45$. On the other hand, no significant effect of lincR-Ccr2-5' AS was found.

\section{Discussion}

Multiple sclerosis (MS) is a chronic disease with neurodegeneration and inflammation comprising a complex immune mechanism [22]. While there is still insufficient information about its exact underlying genetic factors, many studies showed that transcriptional dysregulation may have a role in MS pathogenesis [23].

Studies show the imperative regulatory role of long non-coding RNA (lncRNA) in the immune system and pathogenesis of different CNS disorders, including MS [24]. These factors are vital regulators of CNS function and would be used in the diagnosis and treatment of CNS disorders [25]. LncRNAs are described in different autoimmune, neurodegenerative, and oncologic diseases. The wide variations in their expression throughout innate immune response and during immune cell differentiation determine their directive role in immunity [26]. Therefore, we aimed to investigate, for the first time, the serum-relative expression levels of lincR-Ccr2-5'AS and lncRNA THRIL in patients with RRMS and SPMS.

Our results showed that lincR-Ccr2-5'AS was significantly downregulated in MS patients compared to the controls. There is still insufficient data about lincRCcr2-5' AS in other autoimmune diseases. However, $\mathrm{Hu}$ et al. revealed that lincR-Ccr2-5'AS is expressed in Th2 lineage [27]. It is well-known that Th1 and Th2 lymphocytes and their related cytokines are involved in MS pathogenesis [28]. It was found that the ratio of CD4+ CCR3+/CD4+CCR4+ (which represents Th1/Th2 balance) was higher in MS patients [29], which indicates that there is a shift from Th2 to Th1 in the pathogenesis

Table 2 The fold change of LincR-Ccr2-5'As and THRIL gene expression in MS patient group relative to healthy controls

\begin{tabular}{lll}
\hline Variables & MS cases $(\boldsymbol{n}=\mathbf{7 4})$ mean fold change $\left(\mathbf{2}^{-\Delta \Delta \mathrm{Ct})} \pm \mathrm{SE}\right.$ & $\boldsymbol{p}$-value \\
\hline LincR-Ccr 2-5' AS & $0.43 \pm 0.29$ & $0.03^{*}$ \\
THRIL & $6.18 \pm 2$ & $0.01^{*}$ \\
\hline
\end{tabular}


Table 3 Relative expression levels of LincR-Ccr2-5'As and THRIL based on different clinical characters among cases

\begin{tabular}{|c|c|c|c|c|}
\hline & LincR-Ccr2-5'As, fold change $\left(2^{-\Delta \Delta C t}\right)$, mean \pm SE & $p$-value & THRIL, fold change $\left(2^{-\Delta \Delta C t}\right)$, mean \pm SE & $p$-value \\
\hline \multicolumn{5}{|l|}{ Types } \\
\hline RRMS & $1.08 \pm 0.49$ & 0.2 & $7.91 \pm 2.7$ & 0.04 \\
\hline SPMS & $0.057 \pm 0.24$ & & $1.84 \pm 0.83$ & \\
\hline \multicolumn{5}{|l|}{ Initial presentation } \\
\hline Optic neuritis & $0.622 \pm 0.25$ & 0.001 & $4.91 \pm 4.2$ & $0.01^{\mathrm{a}}$ \\
\hline Sensory & $4.1 \pm 0.91$ & & $1.69 \pm 0.79$ & $0.05^{\mathrm{b}}$ \\
\hline Motor & $0.59 \pm 0.12$ & & $8.1 \pm 2.8$ & $0.001^{c}$ \\
\hline \multicolumn{5}{|l|}{ EDSS score [mean \pm SD] } \\
\hline$E D S S=1-3$ & $0.64 \pm 0.5$ & $0.01^{\mathrm{a}}$ & $6.75 \pm 3.3$ & $0.02^{d}$ \\
\hline$E D S S=3.5-5$ & $1.13 \pm 0.8$ & $0.001^{b, c}$ & $9.5 \pm 4.5$ & $0.001^{e, f}$ \\
\hline EDSS $\geq 5.5$ & $0.11 \pm 0.2$ & & $2.34 \pm 0.9$ & \\
\hline \multicolumn{5}{|l|}{ Treatment } \\
\hline Treatment-naive & $0.17 \pm 0.4$ & $0.001^{d, e, f}$ & $6.84 \pm 5$ & $0.001^{g, h}$ \\
\hline Interferon & $1.45 \pm 0.6$ & & $7.1 \pm 2.8$ & $0.9^{i}$ \\
\hline Immunosuppressants & $0.38 \pm 0.1$ & & 2.81 & \\
\hline
\end{tabular}

${ }^{\mathrm{a} B e t w e e n}$ patients with EDSS $=1-3$ and EDSS $=3.5-5$

${ }^{\mathrm{b}}$ Between patients with EDSS $=1-3$ and EDSS $\geq 5.5$

'Between patients with EDSS $=3.5-5$ and EDSS $\geq 5.5$

${ }^{d}$ Between patients on interferon and immunosuppressant

Between patients on interferon and treatment naive patients

${ }^{\mathrm{f}}$ Between patients on immunosuppressant and treatment naive patients, ${ }^{g, h}$ between treatment naive and patients on immunsuppressant,i between treatment naive and patients on interferon

of MS [29]. Besides, GATA3 "the master regulator of Th2" is the transcription factor for lincR-Ccr2-5'AS in Th2 [8]. It was previously found downregulated in MS patients [30]. Previous studies, which support and explain our findings, showed that downregulation of lincR-Ccr2-5'AS decreases the expression of Ccr1, Ccr3, Ccr2, and Ccr5 genes in Th2 lineage [11]. This affects the enrollment of $\mathrm{T}$ lymphocytes and macrophages into the CNS which was believed to be involved in the pathogenesis of MS [12].

In our study, a higher relative expression level of lincRCcr2-5'AS was reported in patients with sensory manifestations. However, patients with motor manifestations (ataxia) had significantly lower expression levels of lincR-Ccr2-5'AS. To explain this finding, Miller et al. showed that the initial presentation of sensory symptoms is correlated with a decreased risk of conversion of the clinically isolated syndrome (CIS) to MS compared to symptoms that influence movement [31]. A related point to consider is that GATA3 "the master regulator of Th2" is the transcription factor for lincR-

Table 4 Correlation between LincR-Ccr2-5'As and THRIL with other variables among cases

\begin{tabular}{llllll}
\hline Variables among & \multicolumn{2}{l}{ LincR-Ccr2-5'As } & & \multicolumn{2}{l}{ THRIL } \\
\cline { 2 - 3 } \cline { 5 - 6 } cases & $\boldsymbol{r}$ & $\boldsymbol{p}$-value & & $\boldsymbol{r}$ & $\boldsymbol{p}$-value \\
\hline Age (years) & 0.15 & 0.4 & & -0.04 & 0.8 \\
Age of onset (years) & 0.05 & 0.8 & & -0.004 & 0.9 \\
lincR-Ccr2-5'As & -- & -- & & -0.18 & 0.3 \\
\hline
\end{tabular}

Ccr2-5'AS in Th2 [2]. Besides, the GATA3 expression level was found low in MS patients [30]. Fernando et al. found that the overexpression of GATA3 could reduce the severity of disease and delay the onset of Experimental Autoimmune Encephalomyelitis: an animal model for MS (EAE), and with GATA3 overexpression, a few mice had ataxia [32].

We found that the relative expression level of lincRCcr2-5'AS was significantly low in patients with EDSS score $\geq 5$ when compared to those with EDSS $<5$. To explain these results, Khoury et al. showed that the activation of T-lymphocyte is linked to the disease activity in patients with multiple sclerosis [33].

We found a significant increase in the relative expression level of lincR-Ccr2-5' AS after interferon treatment. This increase could be explained by the "steady-state" of chemokine receptor expression (CCR4) in CD4+T-cell surface during treatment with interferon (IFN), since CCR4 mRNA expression is associated with GATA-3 expression [34]. This confers the effect of interferon with the expression of Th2 chemokine receptors in MS patients toward the normalization [34].

Taking these points together, we can assume the potential role of lincR-Ccr2-5AS' in the genetic susceptibility to MS and its impact on the clinical presentation and the disease course.

Our results showed that lncRNA THRIL was upregulated among MS patients. Besides, it was significantly upregulated in patients with RRMS compared to those 


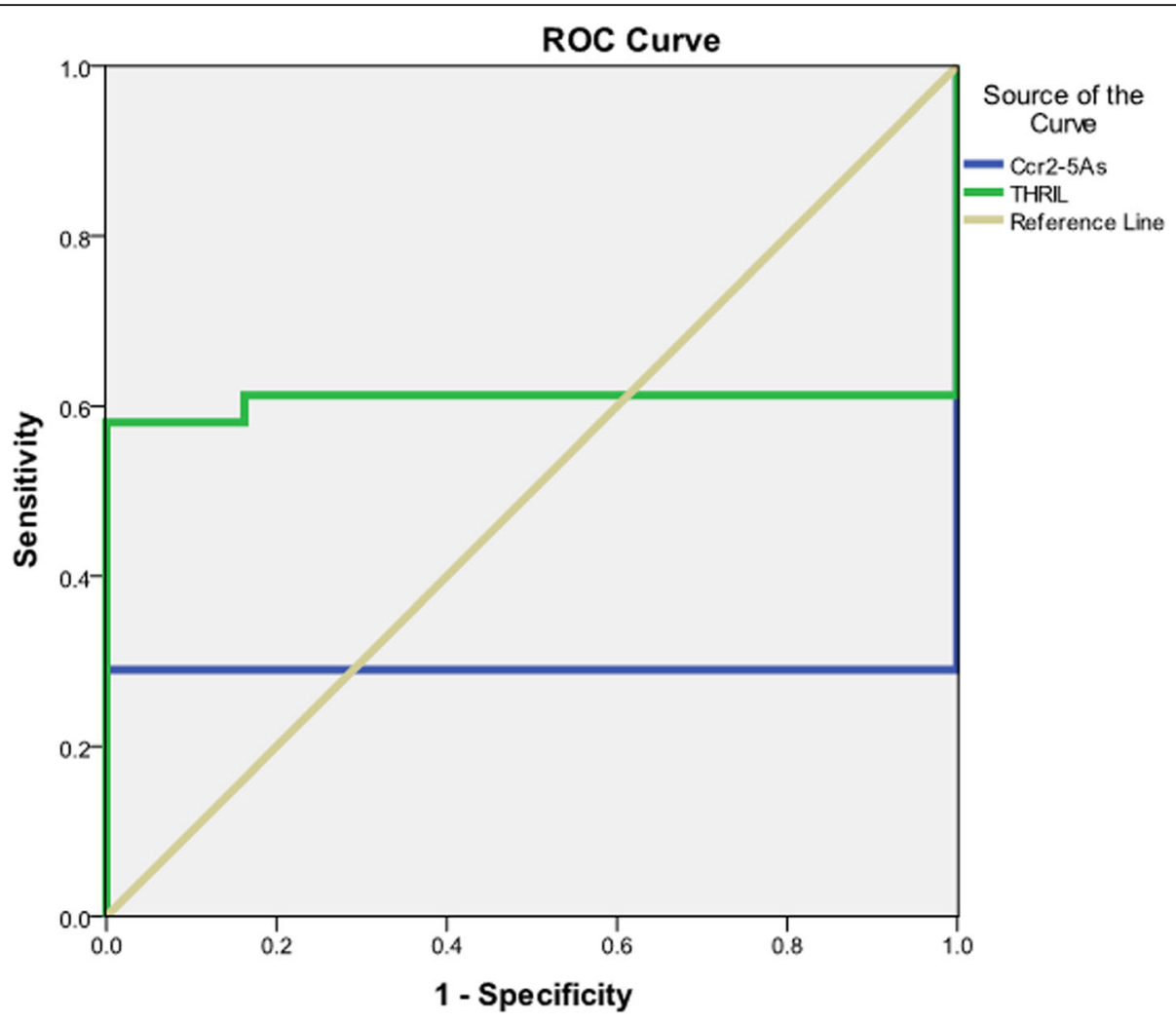

Fig. 3 ROC curve to determine the diagnostic performance of lincR-Ccr2-5'AS and THRIL

with SPMS ( $p=0.04)$. In addition, binary logistic regression showed that THRIL could be a predictor of MS with $p$-value $<0.05, \mathrm{OR}=6.45$. In agreement with our results, Eftekharian et al. [35] detected a significant THRIL upregulation in MS patients. However, they did not find a significant correlation between THRIL expression levels and EDSS. In contrary to our findings, Li et al. showed reduced THRIL expression levels in the acute phase of Kawasaki disease [36]. This contradiction may be attributed to the altered roles of TNF in the pathogenesis of MS and other autoimmune disorders. Besides, it was reported to induce a pro-inflammatory effect in lipopolysaccharide (LPS)-induced osteoarthritis cell injury model [36].

THRIL was demonstrated to control the expression of the innate immune response genes [26]. THRIL is crucial for TNF- $\alpha$ expression. Obradovic et al. demonstrated the role of TNF- $\alpha$ in MS [15]; they found that MS patients had a higher concentration of TNF- $\alpha$ in
CSF and plasma during relapse and SPMS compared to MS patients during remission. The increased TNF- $\alpha$ expression in SPMS may suppress levels of THRIL by the negative feedback inhibition to regulate TNF- $\alpha$ levels expressed by cells in response to inflammatory stimuli [36]. This negative feedback loop could explain the decreased level of THRIL in our SPMS patients. It is wellknown that TNF- $\alpha$ could prompt inflammation, affect the recruitment of the immune cells in tissues, and increase tissue destruction. However, its role in MS needs to be elucidated [37].

Our data showed the potential role of THRIL as a biomarker for genetic susceptibility to MS. Besides, it could have a role as a prognostic biomarker for the prediction of conversion of RRMS to SPMS.

A higher serum level of THRIL was found in patients with EDSS scores $\leq 5$ when compared to those with EDSS $\geq 5.5$. We may attribute the variation of its level among different EDSS groups to the conversion of

Table 5 ROC curve analysis to determine the diagnostic performance of LincR-Ccr2-5'As and THRIL

\begin{tabular}{llllll}
\hline Variable & Sensitivity & Specificity & AUC & Accuracy & Cutoff point \\
\hline LincR-Ccr2-5'As & $71.9 \%$ & $100 \%$ & $71.9 \%$ & $85.9 \%$ & 0.49 \\
THRIL & $57.1 \%$ & $100 \%$ & $57.1 \%$ & $78.6 \%$ & 1.024 \\
\hline
\end{tabular}


Table 6 Logistic regression analysis to determine the effect of LincR-CCr2-5'As and THRIL in prediction of MS

\begin{tabular}{llllll}
\hline Variables & $\boldsymbol{B}$ & S.E. & Wald & $\boldsymbol{P}$ & Exp (B) \\
\hline LincR-Ccr2-5'As & -22.604 & 7234.475 & 0.00 & 0.998 & 0.00 \\
THRIL & 1.865 & 0.806 & 5.362 & $\mathbf{0 . 0 2 1}$ & 6.458 \\
Constant & 0.182 & 0.606 & 0.091 & 0.763 & 1.200 \\
\hline
\end{tabular}

Bold values are significant at $p<0.05$

patients from inflammation to neurodegeneration in a severe state of the disease, which is accompanied by a drop in several inflammatory biomarkers [38].

In our study, we found a significantly higher expression level of THRIL in patients treated with interferon. This high THRIL level might reflect a continuous subclinical immune activity that interferon could not suppress. Despite the role of TNF in MS pathogenesis, TNF inhibitors worsen the disease condition [39]. On the other hand, TNF antagonisms were shown to cause demyelinating lesions. In general, these findings suggest a diverse TNF role in autoimmunity within CNS [39].

This study has some limitations; there were deficient data for both long non-coding RNAs in different autoimmune diseases. Another limitation is the small number of MS patients. Thus, further large-scale studies should be applied. Previous study demonstrated long non-coding RNAs in peripheral blood mononuclear cells in MS patients [9]. We have tried to use another type of samples in order to estimate long non-coding RNAs; comparative studies could be then performed. Finally, due to the nature of case-control studies, some recall and selection bias could not be avoided.

\section{Conclusion}

In conclusion, our study shows that lincR-Ccr2-5' As and THRIL might have a role in genetic susceptibility to MS. Our results demonstrated the downregulation of lincR-Ccr2-5' As and the upregulation of IncRNA THRI L in MS. They may offer good therapeutic targets in the treatment of MS patients. Further studies may be needed to explore the molecular pathways through which lncRNAs influence MS pathogenesis.

\footnotetext{
Abbreviations

CCR: Chemokine receptor; CDNA: Complementary DNA; CIS: Clinically isolated syndrome; CNS: Central nervous system; CSF: Cerebrospinal fluid; Ct: Cycle threshold; EAE: Experimental autoimmune encephalomyelitis; EDSS: Expanded Disability Status Scale; GATA3: GATA-binding protein 3; GAPDH: Glyceraldehyde 3-phosphate dehydrogenase; hnRNPs: Heterogeneous ribonucleoprotein particle; IFN: Interferon; LncRNAs: Long non-coding RNAs; LPS: Lipopolysaccharide; MS: Multiple sclerosis; ncRNA: Non-coding RNA; RA: Rheumatoid arthritis; RRMS: Relapsingremitting multiple sclerosis; RT: Reverse transcription; ROC: Receiver operating characteristic; SPMS: Secondary progressive multiple sclerosis; Th2: T Helper2; THRIL: TNF-A and heterogeneous nuclear ribonucleoprotein L; TNFa: Tumor necrosis factor-alpha
}

\section{Acknowledgements}

We wish to thank Dr. Asmaa Younis for her assistance with the statistics of this manuscript.

\section{Authors' contributions \\ S.R. and O.G. considered the presented idea and carried out the experiment with support from Asmaa Mohammed. S.R. wrote the manuscript. A.H. together with A. M. collected the samples and interpreted the results. All authors provided critical feedback and supported analysis of data. The authors read and approved the final manuscript.}

\section{Funding}

No funding had been received.

\section{Availability of data and materials}

The authors confirm that data supporting the findings of this study are available within the study.

\section{Declarations}

Ethics approval and consent to participate

Written informed consent was provided by all participants. All human studies have been reviewed and approved by the Ethical Committee [committee's reference number, I-211016] at the Faculty of Medicine, Cairo University. It was accomplished by the code of Ethics of the World Medical Association (Declaration of Helsinki) for experiments related to human beings.

\section{Consent for publication}

All authors have approved the manuscript for submission.

\section{Competing interests}

The authors declare that they have no competing interest.

\section{Author details}

${ }^{1}$ Department of Biochemistry and Molecular Biology, Faculty of Medicine, Cairo University, Cairo, Egypt. ${ }^{2}$ Department of Neurology, Faculty of Medicine, Cairo University, Cairo, Egypt. ${ }^{3}$ Department of Medical Biochemistry and Molecular Biology, Faculty of Medicine, Fayoum University, Fayoum, Egypt.

Received: 12 December 2020 Accepted: 23 February 2021

Published online: 29 March 2021

\section{References}

1. Yang X, Wu Y, Zhang B, Ni B (2018) Noncoding RNAs in multiple sclerosis. Clin Epigenetics 10:149

2. Thompson AJ, Baranzini SE, Geurts J, Hemmer B, Ciccarelli O (2018) Multiple sclerosis. Lancet. 391:1622-1636

3. Confavreux C, Vukusic S (2014) The clinical course of multiple sclerosis. Handb Clin Neurol 122:343-369

4. Taheri M, Ghafouri-Fard S, Solgi G, Sayad A, Mazdeh M, Omrani MD (2017) Determination of cytokine levels in multiple sclerosis patients and their relevance with patients' response to Cinnovex. Cytokine. 96:138-143

5. Harris K, Sadiq A (2014) Biomarkers of therapeutic response in multiple sclerosis: current status. Mol Diagn Ther 18:605-617

6. Sánchez Y, Huarte M (2013) Long non-coding RNAs: challenges for diagnosis and therapies. Nucleic Acid Ther 23:15-20

7. Wapinski O, Chang Y (2011) Long noncoding RNAs and human disease. Trends Cell Biol 21:354-361

8. Santoro M, Nociti V, Lucchini M, De Fino C, Losavio FA, Mirabella M (2016) Expression profile of long non-coding RNAs in serum of patients with multiple sclerosis. J Mol Neurosci 59:18-23

9. Zhang F, Gao C, Ma F et al (2016) Expression profile of long noncoding RNAs in peripheral blood mononuclear cells from multiple sclerosis patients. CNS Neurosci Ther 22:298-305

10. Mahad DH, Trapp BD, Lassmann H (2015) Pathological mechanisms in progressive multiple sclerosis. Lancet Neurol 14:183-193

11. Aune TM, Spurlock CF (2016) Long non-coding RNAs in innate and adaptive immunity. Virus Res 26:140-160 
12. Berghmans N, Heremans H, Li S et al (2012) Rescue from acute neuroinflammation by pharmacological chemokine-mediated deviation of leukocytes. J Neuroinflammation 9:243

13. Pegoretti V, Baron W, Laman JD, Eisel ULM (2018) Selective modulation of TNF-TNFRs signaling: insights for multiple sclerosis treatment. Front Immunol 9:925

14. Linkermann A, Stockwell B, Krautwald S, Anders H (2014) Regulated cell death and inflammation: an auto-amplification loop causes organ failure. Nat Rev Immunol 14:759-767

15. Obradovic D, Kataranovski M, Dincic E et al (2012) Tumor necrosis factor-alfa and interleukin-4 in cerebrospinal fluid and plasma in different clinical forms of multiple sclerosis. Vojnosanit Pregl 69:151-156

16. Hawkes CH, Giovannoni G (2010) The McDonald Criteria for multiple sclerosis: time for clarification. Mult Scler 16:566-575

17. Kurtzke JF (1983) Rating neurologic impairment in multiple sclerosis: an expanded disability status scale. Neurology. 33:1444-1452

18. Song B, Li R, Zuo Z et al (2019) LncRNA ENST00000539653 acts as an oncogenic factor via MAPK signalling in papillary thyroid cancer. BMC Cancer 19:297

19. Shaker OG, Senousy MA, Elbaz EM (2017) Association of rs6983267 at 8q24, HULC rs7763881 polymorphisms and serum IncRNAs CCAT2 and HULC with colorectal cancer in Egyptian patients. Sci Rep 7:16246

20. Duan W, Du L, Jiang $X$ et al (2016) Identification of a serum circulating IncRNA panel for the diagnosis and recurrence prediction of bladder cancer. Oncotarget. 7:78850-78858

21. Livak KJ, Schmittgen TD (2001) Analysis of relative gene expression data using real-time quantitative PCR and the 2(-Delta Delta C(T)) method. Methods. 25:402-408

22. Li YH, Yu CY, Li XX et al (2018) Therapeutic target database update 2018: enriched resource for facilitating bench-to-clinic research of targeted therapeutics. Nucleic Acids Res 46:1121-1127

23. Zhang M, Chang Y-C, Shankara S et al (2019) Characterization of the peripheral blood transcriptome in alemtuzumab-treated relapsing-remitting multiple sclerosis patients from the CARE-MS I and II studies (P4. 2-041). Neurology 92:15 Supplement

24. Yin J, Sun W, Li F et al (2020) VARIDT 1.0: variability of drug transporter database. Nucleic Acids Res 48:D1042-D1D50

25. Fenoglio C, Ridolfi E, Galimberti D et al (2013) An emerging role for long non-coding RNA dysregulation in neurological disorders. Int J Mol Sci 14: 20427-20442

26. Zhang Y, Cao X (2016) Long noncoding RNAs in innate immunity. Cell Mol Immunol 13:138-147

27. Hu G, Tang Q, Sharma S et al (2013) Expression and regulation of intergenic long noncoding RNAs during T cell development and differentiation. Nat Immunol 11:1190-1198

28. Cravens PD, Kieseier BC, Hussain R et al (2013) The neonatal CNS is not conducive for encephalitogenic Th1 T cells and B cells during experimental autoimmune encephalomyelitis. J Neuroinflammation 10:67

29. Nakajima H, Fukuda K, Doi Y et al (2004) Expression of TH1/TH2-related chemokine receptors on peripheral T cells and correlation with clinical disease activity in patients with multiple sclerosis. Eur Neurol 52:162-168

30. Edstrom M, Mellergard J, Mjosberg J et al (2011) Transcriptional characteristics of CD4 T cells in multiple sclerosis: relative lack of suppressive populations in blood. Mult Scler 17:57-66

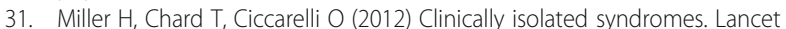
Neurol 11:157-169

32. Fernando V, Omura S, Sato F et al (2014) Regulation of an autoimmune model for multiple sclerosis in Th2-biased GATA3 transgenic mice. Int J Mol Sci 15:1700-1718

33. Khoury SJ, Guttmann CR, Orav EJ, Kikinis R, Jolesz FA, Weiner HL (2000) Changes in activated T cells in the blood correlate with disease activity in multiple sclerosis. Arch Neurol 57:1183-1189

34. Krakauer M, Sorensen PS, Khademi M, Olsson T, Sellebjerg F (2006) Dynamic T-lymphocyte chemokine receptor expression induced by interferon-beta therapy in multiple sclerosis. Scand J Immunol 64:155-163

35. Eftekharian M, Ghafouri S, Soudyab M et al (2017) Expression analysis of long non-coding RNAs in the blood of multiple sclerosis patients. J Mol Neurosci 63:333-341

36. Li Z, Chao TC, Chang KY et al (2014) The long noncoding RNA THRIL regulates TNFa expression through its interaction with hnRNPL. Proc Natl Acad Sci U S A 111:1002-1007
37. Liu G, Wang Y, Zhang M et al (2019) Long non-coding RNA THRIL promotes LPS-induced inflammatory injury by down-regulating microRNA-125b in ATDC5 cells. Int Immunopharmacol 66:354-361

38. Kalliolias GD, Ivashkiv LB (2016) TNF biology, pathogenic mechanisms and emerging therapeutic strategies. Nat Rev Rheumatol 12:49-62

39. Robinson WH, Genovese MC, Moreland LW (2001) Demyelinating and neurologic events reported in association with tumor necrosis factor alpha antagonism: by what mechanisms could tumor necrosis factor alpha antagonists improve rheumatoid arthritis but exacerbate multiple sclerosis? Arthritis Rheum 44:1977-1983

\section{Publisher's Note}

Springer Nature remains neutral with regard to jurisdictional claims in published maps and institutional affiliations.

\section{Submit your manuscript to a SpringerOpen ${ }^{\circ}$ journal and benefit from:}

- Convenient online submission

- Rigorous peer review

- Open access: articles freely available online

- High visibility within the field

- Retaining the copyright to your article

Submit your next manuscript at $>$ springeropen.com 